\title{
Spatial Loading in V-BLAST Systems with Limited Feedback and ZF-OSIC Detection
}

\author{
Maurizio Magarini \\ Departments di Elettronica e Informazione, Politecnico di Milano, \\ Piazza Leonardo da Vinci, 32, I-20133 Milano (Italy) \\ magarini@elet.polimi.it
}

\begin{abstract}
In this paper we propose a new spatial loading scheme for the Vertical Bell Layered Space Time (V-BLAST) system where the detection of symbols belonging to the same transmitted vector is done by zero-forcing with ordered successive interference cancellation (ZF-OSIC). Assuming no error propagation, the ZF-OSIC detection process decouples the channel matrix into a set of independent parallel sub-channels. In our proposal the data rate and the average power to be allocated on each sub-channel are obtained by minimizing the asymptotic approximation of the average bit error rate for the given desired total data rate and the total average transmitted power. The feedback sent from the receiver to the transmitter consists of the average signal-to-noise ratio and the detection order. Computer simulations are used to demonstrate the effectiveness of our proposal over the conventional V-BLAST.
\end{abstract}

\section{INTRODUCTION}

The ever increasing demand for high spectral efficiency in wireless communications has contributed to consider the introduction of architectures based on multiple antenna elements both for the transmitter and for the receiver [1]. The Vertical Bell Layered Space Time (V-BLAST) system, originally proposed in [2], is one of the most investigated architectures to achieve this higher spectral efficiency. In such a scheme the input data sequence is first demultiplexed in $N_{T}$ sub-streams and then transmitted, over the multiple input multiple output (MIMO) channel, through $N_{T}$ antenna elements. At the receiver, an estimate of the transmitted data symbols is produced by processing the signals received from $N_{R} \geq N_{T}$ antenna elements. The performance of the $\mathrm{V}$ BLAST architecture, in terms of error probability, is strongly dependent on the technique implemented in the receiver to detect the $N_{T}$ transmitted sub-streams. In this paper we focus on a V-BLAST system where the detection is done by zeroforcing ${ }^{1}$ with ordered successive interference cancellation (ZFOSIC). The introduction of an optimal ordering, based on the maximization of the minimum post-detection SNR over all possible detection orderings, improves the performance over the fixed-order successive interference cancellation (SIC) [2].

In the implementation of the conventional V-BLAST the total data rate and the total average transmitted power are equally divided among the transmit antenna elements. This equal data rate and equal average power allocation strategy

${ }^{1} \mathrm{~A}$ better performance is obtained with the minimum-mean square error OSIC detection. Since its analytical performance evaluation is complicated we do not consider it in the present work. is the optimal solution when channel state information (CSI) is not available at the transmitter [3]. When CSI is present, spatial loading algorithms, assigning the data rate and the average power on each sub-channel, allow us to obtain a performance improvement both for the case of ZF-SIC and ZF-OSIC detection [4]-[11].

Spatial loading in V-BLAST systems with ZF-SIC detection has been considered in [5]-[6] with the aim of minimizing the average probability of symbol or vector error. While the approach considered in [5] allocates only the average powers across transmitters, in [6] both the data rates and the average powers are optimized. Note that, an important characteristic of the two approaches is that of requiring a very limited feedback from the receiver consisting only of the average SNR. The spatial loading problem in V-BLAST systems with ZF-OSIC detection has been investigated in [7]-[10] for the case of power allocation only. A spatial loading algorithm that assigns the data rate and the average power to each transmit antenna so that the instantaneous probabilities of symbol errors are equalized on all the parallel sub-channels has been proposed in [11]. The feedback required at the transmitter by such a scheme consists of the data rate and the average power associated to each transmitter.

In this paper we consider the optimization of the average bit error rate (BER) for a fixed total data rate and a given total average transmitted power. The resulting spatial loading scheme assigns predetermined data rates and average powers to transmit antenna elements considering a very limited feedback from the receiver that consists of the average SNR and the order in which the sub-streams are detected. As in [5], [6], and [10], the proposed scheme introduces a simplification compared to the other approaches where the information sent by the receiver consists of the average power or the data rate and the average power to be allocated on each transmitter.

The paper is organized as follows. The model of the system we focus on is described in section II. The proposed spatial loading scheme is introduced in section III. Experimental results are shown in section IV and, finally, conclusions are drawn in section $\mathrm{V}$.

\section{SYSTEM MODEL}

Let $\mathbf{a}=\left[a_{1}, a_{2}, \ldots, a_{N_{T}}\right]^{T}$ denote the $N_{T} \times 1$ vector of independent transmitted QAM symbols each having unitary average power ( ${ }^{T}$ denotes transposition). We assume that the 
complex symbol $a_{n}$, transmitted from the $n$-th antenna, is drawn from a square QAM constellation with $M_{n}$ points. The received signal vector at a particular time instant is represented in complex baseband form as

$$
\mathbf{r}=\mathbf{H} \mathbf{P}^{1 / 2} \mathbf{a}+\mathbf{w}
$$

where $\mathbf{H}$ is the $N_{R} \times N_{T}$ MIMO channel matrix whose elements are i.i.d. random variables (RVs) having uniformdistributed phase and Rayleigh-distributed magnitude with average power equal to $1, \mathbf{P}=\operatorname{diag}\left[p_{1}, \ldots, p_{N_{T}}\right]$ is a diagonal matrix whose $n$-th entry $p_{n}$ denotes the average power radiated from the $n$-th antenna and $\mathbf{w}$ is the $N_{R} \times 1$ noise vector of i.i.d. complex Gaussian RVs with zero mean and variance $\sigma_{w}^{2}$. The value of $p_{n}$ is assigned to transmitter $n$ on the basis of the feedback sent by the receiver. The constraint $\operatorname{trace}(\mathbf{P})=1$ is set on the total average transmitted power. We assume that both $\mathbf{H}$ and $\sigma_{w}^{2}$ are perfectly estimated at the receiver and that the feedback channel is error free. The average SNR is defined as $\bar{\gamma} \triangleq 1 / \sigma_{w}^{2}$.

As in [7]-[10], the ZF-OSIC detection process at the receiver coincides with that implemented in the conventional V-BLAST, that is, based on the ordered post-detection SNR obtained when $\mathbf{P}=\frac{1}{N_{T}} \mathbf{I}_{N_{T}}\left(\mathbf{I}_{k}\right.$ denotes the $k \times k$ identity matrix). Therefore, the detection order is not influenced by the transmit power allocation.

\section{The Proposed Spatial LoAding Scheme}

Assuming no error propagation, a detection process based either on ZF-SIC or ZF-OSIC decouples the channel matrix into a set of independent parallel channels with increasing diversity order, $N_{R}-N_{T}+i$ being the diversity order at the $i$-th processing step [12]. According to this view, we can apply rate and power loading strategies that have been devised to optimize different performance criteria in case of transmission over independent parallel channels where CSI is available at the transmitter (see [13] and references therein).

In particular, we are interested in the minimization of the average BER when the detection is done by ZF-OSIC subject to the constraints of a fixed total data rate and a total average transmitted power. The total average BER is given by

$$
\bar{P}_{b}=\frac{1}{b} \sum_{i=1}^{K} b_{i} \bar{P}_{b ; i}
$$

where $\bar{P}_{b ; i}$ denotes the average BER at $i$-th detection step, $b_{i}=$ $\log _{2} M_{i}$ is the data rate (number of bits) associated to the substream detected in the same step and $K$, with $1 \leq K \leq N_{T}$, is the number of sub-streams used at the transmitter to obtain the desired fixed data rate

$$
b=\sum_{i=1}^{K} b_{i}
$$

It is worth noting that the use of a number of transmitted substreams equal to $K$, lower than $N_{T}$, implies an increase of the diversity order at the $i$-th processing step to $N_{R}-K+i$.
Neglecting the effects of error propagation, $\bar{P}_{b ; i}$ can be written as a function of the average SNR per channel $\bar{\gamma}_{c ; i}$ and the number of QAM constellation points $M_{i}$. The average SNR per channel at $i$-th step is defined as

$$
\bar{\gamma}_{c, i}\left(\alpha_{i}\right) \triangleq \alpha_{i} / \sigma_{w}^{2}, \quad i=1, \ldots, K
$$

where $\alpha_{i}$ denotes the average transmitted power allocated to the transmitted sub-stream detected in such a step. Average powers are assigned to transmit antenna elements according to the detection ordering communicated by the receiver, that is $p_{k_{i}}=\alpha_{i}$, where $k_{i} \in\{1, \ldots, K\}$ represents the integer identifying the transmitted sub-stream to be detected at the $i$ th step. The average BER at $i$-th detection step is calculated as

$$
\bar{P}_{b ; i}\left(M_{i}, \bar{\gamma}_{c ; i}\right)=\int_{0}^{\infty} P_{b ; i}\left(M_{i}, \gamma_{c ; i}\right) f_{i}\left(\gamma_{c ; i}\right) d \gamma_{c ; i},
$$

where the instantaneous BER $P_{b ; i}\left(M_{i}, \gamma_{c ; i}\right)$ is averaged over the probability density function (pdf) $f_{i}\left(\gamma_{c ; i}\right)$ of the instantaneous SNR $\gamma_{c ; i}$.

Our spatial loading scheme is based on the minimization of

$$
\min _{\alpha_{1}, \cdots, \alpha_{K}} \frac{1}{b} \sum_{i=1}^{K} b_{i} \bar{P}_{b ; i}\left(M_{i}, \alpha_{i}\right)
$$

subject to

$$
\sum_{i=1}^{K} \alpha_{i}=1
$$

Note that in (6) the dependence of $\bar{P}_{b ; i}\left(M_{i}, \bar{\gamma}_{c ; i}\right)$ on $\alpha_{i}$ has been made explicit by using (4). The minimization of (6) gives the optimal powers $\alpha_{i}$ for the given average SNR and the given assignment of $M_{i}, i=1, \ldots, K$. The sum of the data rates on the $K$ used sub-channels must be kept constant to satisfy the constraint on the total data rate given in (3).

In order to compute the average BER given in (5) for each detection step, we consider as instantaneous BER the approximation proposed in [14] for square $M$-QAM constellations

$$
P_{b}(M, \gamma) \approx \frac{1}{5} \exp (-g \gamma)
$$

where the constant $g$ is defined as

$$
g=\frac{3}{2(M-1)} .
$$

The exact analytical expression of the pdfs $f_{i}\left(\gamma_{c ; i}\right)$ is known only for the case of two transmit antennas and an arbitrary number of receive antennas [3], [12]. However, the use of these two pdfs in (5) would lead to an average BER that does not lend itself to a manageable form from an optimization point of view. For this reason we consider their asymptotic approximations that are valid at high SNR. For an $N_{R} \times 2$ MIMO system the asymptotic approximation of the pdf at the first detection step is given by

$$
\begin{aligned}
& f_{1}\left(\gamma_{c ; 1}\right) \approx \frac{2}{\left(N_{R}-2\right) ! \bar{\gamma}_{c ; 1}^{N_{R}-1}} \gamma_{c ; 1}^{N_{R}-2} \\
& \quad \cdot\left[\exp \left(-\frac{\gamma_{c ; 1}}{\bar{\gamma}_{c ; 1}}\right)-\exp \left(-\frac{2 \gamma_{c ; 1}}{\bar{\gamma}_{c ; 1}}\right)\left(1-2^{-N_{R}}\right)\right]
\end{aligned}
$$


while that at the second is

$$
f_{2}\left(\gamma_{c ; 2}\right) \approx \frac{2}{\left(N_{R}-1\right) ! \bar{\gamma}_{c ; 2}^{N_{R}}} \gamma_{c ; 2}^{N_{R}-1} \exp \left(-\frac{2 \gamma_{c ; 1}}{\bar{\gamma}_{c ; 1}}\right) .
$$

The resulting asymptotic approximations of the average BERs at the first and second detection steps are respectively

$$
\bar{P}_{b ; 1}\left(M_{1}, \bar{\gamma}_{c ; 1}\right) \approx \frac{2^{1-N_{R}}}{5\left(g_{1} \bar{\gamma}_{c ; 1}\right)^{N_{R}-1}}
$$

and

$$
\bar{P}_{b ; 2}\left(M_{2}, \bar{\gamma}_{c ; 2}\right) \approx \frac{2}{5\left(g_{2} \bar{\gamma}_{c ; 2}\right)^{N_{R}}}
$$

where $g_{1}$ and $g_{2}$ are computed from (9) according to the values of $M_{1}$ and $M_{2}$. Considering the analysis developed in [3], [12], and [15], it is possible to write the analytic asymptotic approximations of the pdfs at the different detection steps up to $N_{T}=4$ and $N_{R}=4$. In order to derive their analytic asymptotic approximations at high SNR, in [10] we have extended the analysis developed in [3], [12] for the case of two transmit antennas by taking into account the conjectures used in [15] to analyze the asymptotic outage probability for $N_{R}=N_{T}=3,4$. The asymptotic approximations of the average BERs at the different detection steps can be obtained in the same way we have computed the expressions of the symbol error probabilities at the different detection steps in [10] and by considering that now the instantaneous BER is given by (8).

The data rate and the average power to be assigned on each sub-channel, for a given average SNR, are obtained considering the minimum of the average BER among those achieved by different combinations of data rates on the sub-channels for all the possible values of $K$. The average powers are allocated to different transmit antenna elements on the basis of the average SNR and the detection order communicated by the receiver. As in [9], to find the minimum of (6) we have used the MATLAB function FMINCON. This function attempts to find the constrained minimum of a scalar function of several variables starting at an initial estimate.

\section{NUMERICAL RESUltS}

In this section we present some simulation results where we compare the performance of the proposed spatial loading algorithm with that of the conventional V-BLAST. We assume that the number of receive antennas is equal to the number of transmit antennas, that is $N_{R}=N_{T}$. Fig. 1 reports the performance comparison between our proposed spatial loading scheme and the conventional V-BLAST for the $2 \times 2$ case and a total data rate of 8 bits per channel use. For this situation we have found that the asymptotic approximation of the average BER provides a good estimate of the true performance in the average SNR region defined by $\bar{\gamma} \geq 15 \mathrm{~dB}$. In the conventional V-BLAST a data rate of 4 bits per channel use is allocated on each spatial sub-channel. For the proposed spatial loading scheme the optimization provides a number of $K=2$ used sub-channels in the average SNR region from 15 to $45.5 \mathrm{~dB}$ and $K=1$ for higher values of the average SNR. In the

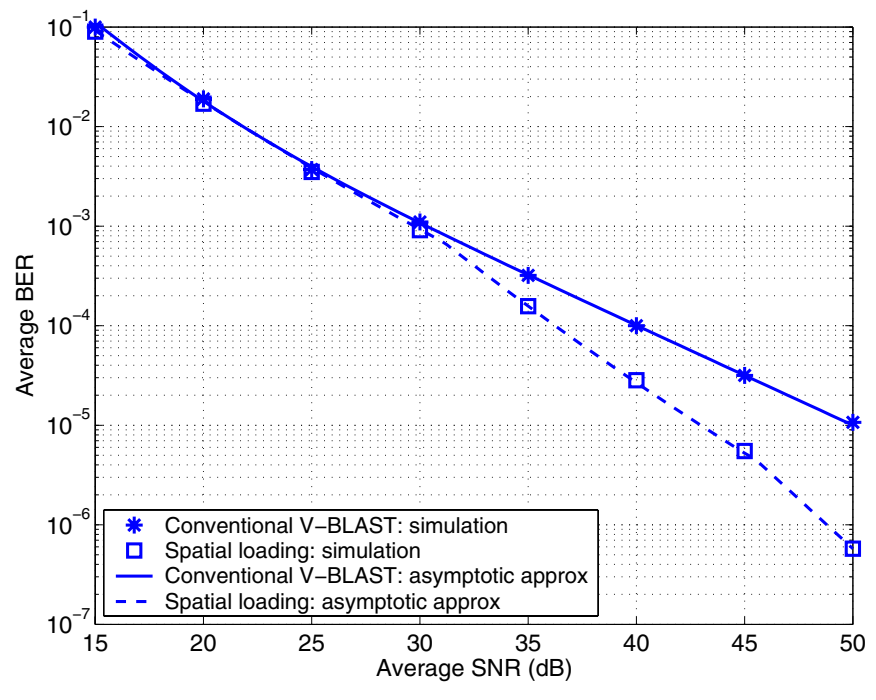

Fig. 1. Average BER comparison between the conventional V-BLAST and the proposed spatial loading scheme for a $2 \times 2$ MIMO system with a total data rate of 8 bits channel use.

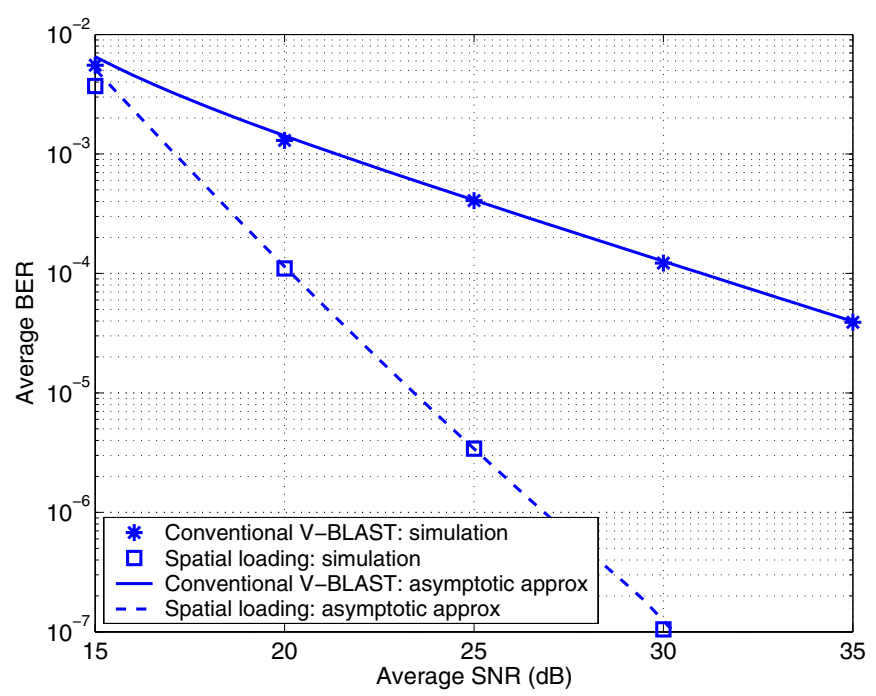

Fig. 2. Average BER comparison between the conventional V-BLAST and the proposed spatial loading scheme for a $3 \times 3$ MIMO system with a total data rate of 6 bits channel use.

average SNR region from 15 to $45.5 \mathrm{~dB}$ the data rate on both the sub-channels is 4 bits per channel use up to $31.2 \mathrm{~dB}$ while it is of 2 and 6 bits per channel use, respectively, for the first and the second sub-stream in the average SNR region from $31.2 \mathrm{~dB}$ to $45.5 \mathrm{~dB}$. These changes in the values of $K$ and data rates are visible as modifications of the slope of the curve giving the average BER in Fig. 1. Note that, in this case the performance for an average SNR greater than $45.5 \mathrm{~dB}$ coincides with that obtained considering the transmission of a 256-QAM where at the receiver we perform maximal ratio combining with two antennas. The figure shows that at $10^{-4}$ the proposed spatial loading scheme allows us to obtain a gain of $3.7 \mathrm{~dB}$ over the conventional V-BLAST. The gain of the spatial loading scheme increases at a higher SNR due to the higher slope of 


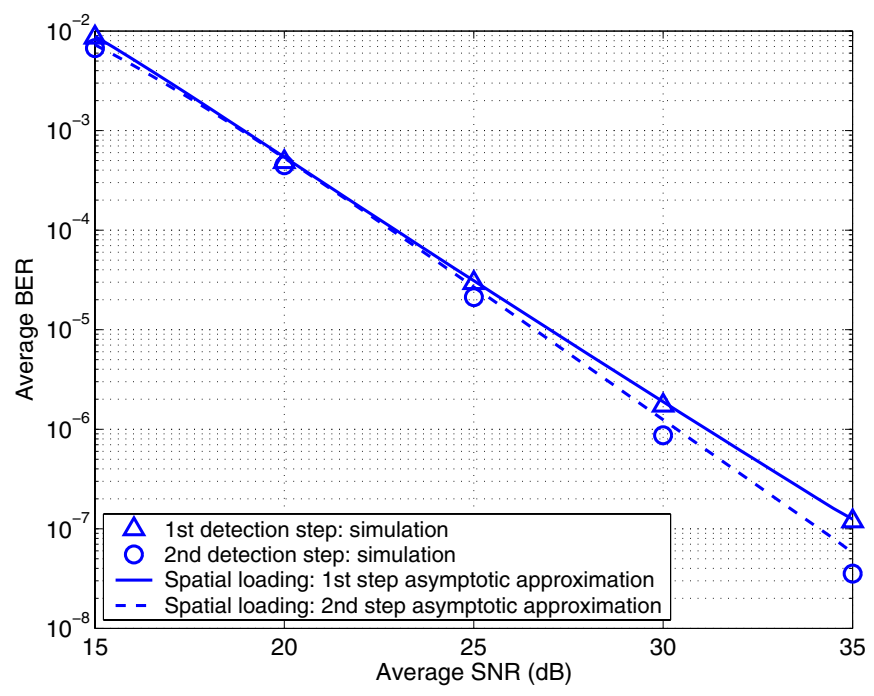

Fig. 3. Average BERs at the first and the second detection steps for the proposed spatial loading scheme for a $3 \times 3$ MIMO systems with a total data rate of 6 bits channel use.

its average BER curve compared to that of the conventional approach. In order to validate the asymptotic approximation, numerical results obtained by Monte Carlo (MC) simulations are also reported in Fig. 1. We observe that no differences exist between the asymptotic approximation and MC simulations.

Fig. 2 shows the results obtained for the $3 \times 3$ case and a total data rate of 6 bits per channel use. Also for this case no differences have been observed between the asymptotic approximations and MC simulations in the region $\bar{\gamma} \geq 15 \mathrm{~dB}$. At $10^{-4}$ the proposed spatial loading scheme gives a gain of $11.3 \mathrm{~dB}$ compared to the conventional one. The superior gain obtained for $N=3$ compared to $N=2$ can be easily explained by observing that the optimization of (6) in this case gives $K=2$ with a data rate of 2 and 4 bits per channel use on the first and the second detected sub-channel, respectively. Hence, the huge difference of performance between the two curves reported in the figure is given by their different slopes in the considered average SNR region. The average BERs obtained by the proposed spatial loading scheme at the first and second detection steps are reported in Fig. 3. As the figure shows, the proposed spatial loading scheme tends to equalize the average BERs at the different detection steps. Again, we obtain a good agreement between theoretical and simulation values.

Numerical results for the $4 \times 4$ case with the same total data rate of the $2 \times 2$ case are reported in figure 4 . We observe that at $10^{-4}$ the gain achieved by the proposed spatial loading scheme over the conventional one is similar to that obtained in the $3 \times 3$ case. In the average SNR region from 15 to $30.2 \mathrm{~dB}$ the optimization of (6) gives $K=3$ with a data rate of 2 bits per channel use on the first detected sub-channel, 4 bits per channel use on the second detected sub-channel and 4 bits per channel use on the last detected sub-channel. For an average SNR between 30.2 and $35 \mathrm{~dB}$ the optimization gives $K=2$ with 4 bits per channel used on both the sub-channels.

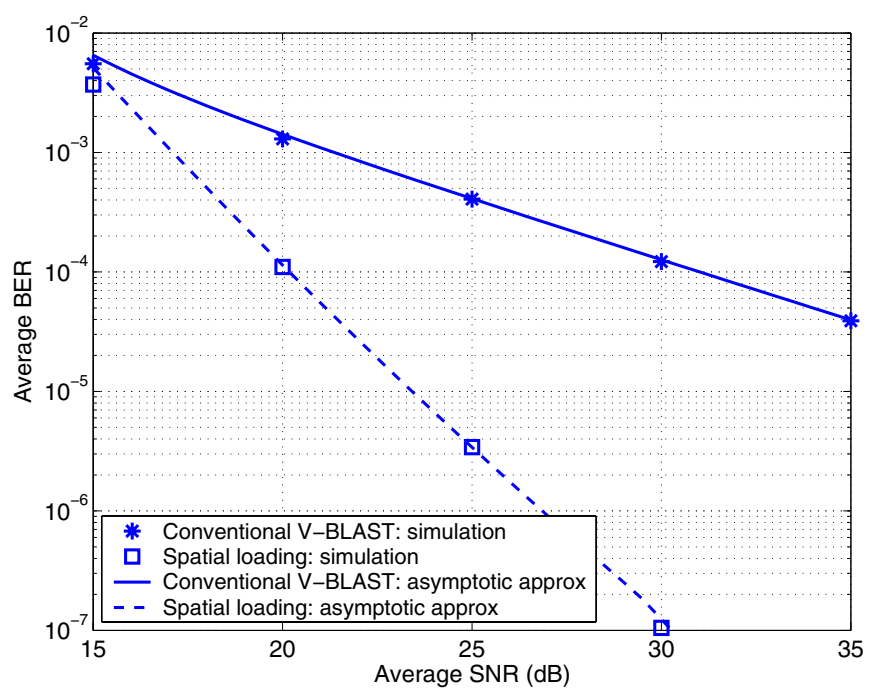

Fig. 4. Average BER comparison between the conventional V-BLAST and the proposed spatial loading scheme for a $4 \times 4$ MIMO system with a total data rate of 8 bits per channel use.

For all the examples we emphasize that in the region $\bar{\gamma}<15$ $\mathrm{dB}$ the asymptotic approximation does not provide a good estimate of the true performance. In this region the proposed spatial loading scheme would not provide a gain compared to the conventional one due to the lack of validity of the asymptotic approximations.

\section{CONCLUSIONS}

This paper presents a new spatial loading scheme for $\mathrm{V}$ BLAST systems with ZF-OSIC detection based on the minimization of the asymptotic approximation of the average BER. The data rate and the power allocation at the transmitter is done on the basis of a very limited feedback from the receiver that consists only in the average SNR and the detection order. Simulation results have been used to demonstrate the effectiveness of the proposed spatial loading approach compared to the conventional equal power allocation scheme for a $\mathrm{V}$ BLAST system with a maximum of four transmit and receive antenna elements.

\section{REFERENCES}

[1] A. Paulraj, R. Nabar, D. Gore, "Introduction to space-time wireles communications," Cambridge, UK: Cambridge University Press, 2003.

[2] P. W. Wolniansky, G. J. Foschini, G. D. Golden, R. A. Valenzuela, "VBLAST: an architecture for realizing very high data rates over the richscattering wireless channel," in Proc. ISSSE, Pisa, Italy, pp. 295-300, 29 Sept.-2 Oct. 1998

[3] R. T. Xu, C. M. Lau, "Analytical approach of V-BLAST performance with two transmit antennas," in Proc. WCNC, New Orleans, LA, Mar. 2005.

[4] C. Windpassinger, Detection and Precoding for Multiple Input Multiple Output Channels. Ph.D. dissertation, Universität Erlangen- Nërnberg, 2004.

[5] V. Kostina, S. Loyka, "On optimization of the V-BLAST algorithm," IEEE Proc. IZS, Zurich, Switzerland, pp. 110-113, Feb. 2006.

[6] N. Prasad, M. K. Varanasi, "Analysis of decision feedback detection for MIMO Rayleigh-fading channels and the optimization of power and rate allocations," IEEE Trans. Inform. Theory, vol. 50, pp. 1009-1025, June 2004. 
[7] S. H. Nam, Oh-S. Shin, K. B. Lee, "Transmit power allocation for a modified V-BLAST system," IEEE Trans. Commun., vol. 52, pp. 10741079, July 2004.

[8] N. Wang, S. D. Blostein,"Minimum BER power allocation for MIMO spatial multiplexing systems," in Proc. ICC, Seoul, Korea, pp. 22822286, May 2005.

[9] R. Kalbasi, D. D. Falconer, A. H. Banihashemi, "Optimum power allocation for a V-BLAST system with two antennas at the transmitter,' IEEE Commun. Lett., vol. 9, pp. 826-828, Sept. 2005.

[10] M. Magarini, "Transmit Power Allocation for V-BLAST Systems with ZF-OSIC Detection," in Proc. ISWCS, Valencia, Spain, pp. 11-15, Sept. 2006.

[11] T. Vencel, C. Windpassinger, R. F. H. Fischer, "Sorting in the V-BLAST algorithm and loading," in Proc. CSN, Malaga, Spain, pp. 304-309, Sept. 2002.

[12] S. Loyka, F. Gagnon, "Performance analysis of the V-BLAST algorithm: an analytical approach," IEEE Trans. Wireless Commun., vol. 3, pp. 1326-1337, July 2004.

[13] T. Hunziker, D. Dahlhaus, "Optimal power allocation for OFDM systems with ideal bit-interleaving and hard decision decoding," in Proc. ICC, Anchorage, AK, pp. 3392-3397, May 2003.

[14] A. J. Goldsmith, S. G. Chua, "Variable-rate variable-power MQAM for fading channels," IEEE Trans. Commun., vol. 45, pp. 1218-1230, Oct. 1997.

[15] S. Loyka, F. Gagnon, "Analytical framework for outage and BER analysis of the V-BLAST algorithm," IEEE Proc. IZS, Zurich, Switzerland, pp. 120-123, Feb. 2004. 